\title{
Multiplex ligation-depending probe amplification is not suitable for detection of low-grade mosaicism
}

\author{
Monique M van Veghel-Plandsoen ${ }^{\star 1}$, Cokkie H Wouters $^{1}$, Joan NR Kromosoeto ${ }^{1}$, \\ Mariska C den Ridder-Klünnen ${ }^{1}$, Dicky JJ Halley ${ }^{1}$ and Ans MW van den Ouweland ${ }^{1}$
}

\begin{abstract}
'Apparent non-penetrance' occurs in several genetic disorders, including tuberous sclerosis complex and neurofibromatosis type 1: clinically unaffected parents may have multiple affected offspring. Germ line or somatic mosaicism in one of the parents of the index patient is the probable cause and results in an enhanced recurrence risk. Therefore, it is of great importance to use the most sensitive technology for testing DNA of the parents of the index patient for the presence/absence of the familial mutation. To detect large rearrangements multiplex ligation-depending probe amplification (MLPA) is often used. Here we show that MLPA is less sensitive in detecting low-grade somatic mosaicism than fluorescence in situ hybridization (FISH) or a mutation-specific PCR test. Therefore, we recommend FISH (if possible) or PCR analysis for the analysis of parental DNA. European Journal of Human Genetics (2011) 19, 1009-1012; doi:10.1038/ejhg.2011.60; published online 13 April 2011
\end{abstract}

Keywords: sensitivity; MLPA; mosaicism; FISH; mutation-specific PCR test; recurrence risk

\section{INTRODUCTION}

Several genetic disorders, including tuberous sclerosis complex (TSC) and neurofibromatosis type 1 (NF1), show the effect of clinically unaffected parents who have multiple affected offspring. Germ line or somatic mosaicism in one of the parents has been described, resulting in a recurrence risk of up to $50 \%{ }^{1}$

TSC is an autosomal dominant disorder, caused by a mutation in either TSC1 or TSC2, inactivating the protein. ${ }^{2}$ About $70-80 \%$ of the TSC patients are sporadic cases. ${ }^{3}$ This observation does not exclude germ line mosaicism in one of the parents.

$\mathrm{NF} 1$ is an autosomal dominant neurocutaneous disorder, ${ }^{4}$ caused by a mutation in $N F 1$, resulting in an inactive protein.

Point mutations as well as large rearrangements occur in TSC1, TSC2 and NF1. For the detection of large rearrangements, multiplex ligation-dependent probe amplification (MLPA) analysis is often used. ${ }^{5,6}$ No data about the sensitivity of MLPA analysis are available. Here we present data that MLPA is not suitable for the detection of low-grade mosaicism. We recommend confirmation of an abnormal MLPA pattern by using either a mutation-specific PCR test after characterization of the breakpoint or, if possible, by fluorescent in situ hybridization (FISH) analysis. These methods can be used for analysis of other family members, especially the parents, for a better risk assessment.

\section{MATERIALS AND METHODS}

MLPA and FISH analysis

MLPA analysis was performed according to the manufacturer's instructions. Products were run on an automated sequencer (ABI 3730XL, Applied Biosystems, Foster City, CA, USA) and data were analyzed using Genemarker version 1.5 (Softgenetics, State College, PA, USA). In our routine diagnostic setting, the analysis is based on the MLPA ratio. Quantification is based on the peak height and normalization uses an internal control probe. For sequences present in two copies in the sample, the resulting value is 1.0. A reduction or increase in peak height values results in values $<0.70$ or $>1.30$, respectively (indicating a deletion or duplication). The kits used were: SALSA P046 for TSC2 and SALSA P081/P082 for NF1 (MRC-Holland BV, Amsterdam, The Netherlands).

Because of the possibility of somatic mosaicism, at least 50 metaphases derived from cultured PHA-stimulated peripheral blood lymphocytes were analyzed when performing FISH analysis for NF1 using the probe cP5. ${ }^{7}$

Characterization and confirmation of the breakpoint in TSC family Using long-range PCR analysis, the breakpoints of an observed MLPA abnormality were characterised by sequencing the aberrant PCR product. A gene defect-specific PCR test was developed using three primers, one common primer, one primer specific for the gene defect and one primer more $3^{\prime}$ of the defect creating a wild-type fragment that is larger than the mutant fragment. In this way, detection of the mutant signal is favored (all primer sequences are available on request). The PCR products were run on an agarose gel.

\section{RESULTS AND DISCUSSION}

Previously, an abnormal pattern using Southern blot analysis with CDNA probes of TSC2 was obtained in the index patient of family 9703. ${ }^{1}$ DNA of the father showed a faint band at the same position of the abnormal fragment in DNA of the index patient, indicating that he was a somatic mosaic for his daughter's gene defect.

MLPA of TSC2 showed a pattern consistent with a duplication of exon 15-exon 26 (a probe for exon 27 is not present in the MLPA kit; Figure 1c). Using our routine MLPA protocol, the duplication was hardly detectable in DNA of the father (Figure 1b). Using a range of paternal input DNA of 25-250 ng DNA (Figure 1j-1), no clearly visible duplication could be shown. Using the internal control probe normalization as well as the population normalization using peak height as well as peak area calculation, also normalization and quantification of

\footnotetext{
${ }^{1}$ Department of Clinical Genetics, Erasmus Medical Center, Rotterdam, The Netherlands

*Correspondence: MM van Veghel-Plandsoen, Department of Clinical Genetics, Erasmus Medical Center, PO Box 2040, Rotterdam 3000 CA, The Netherlands.

Tel: +31 107043197; Fax: +31 107044764;
}

E-mail: m.plandsoen@erasmusmc.nl

Received 4 January 2011; revised 1 March 2011; accepted 4 March 2011; published online 13 April 2011 

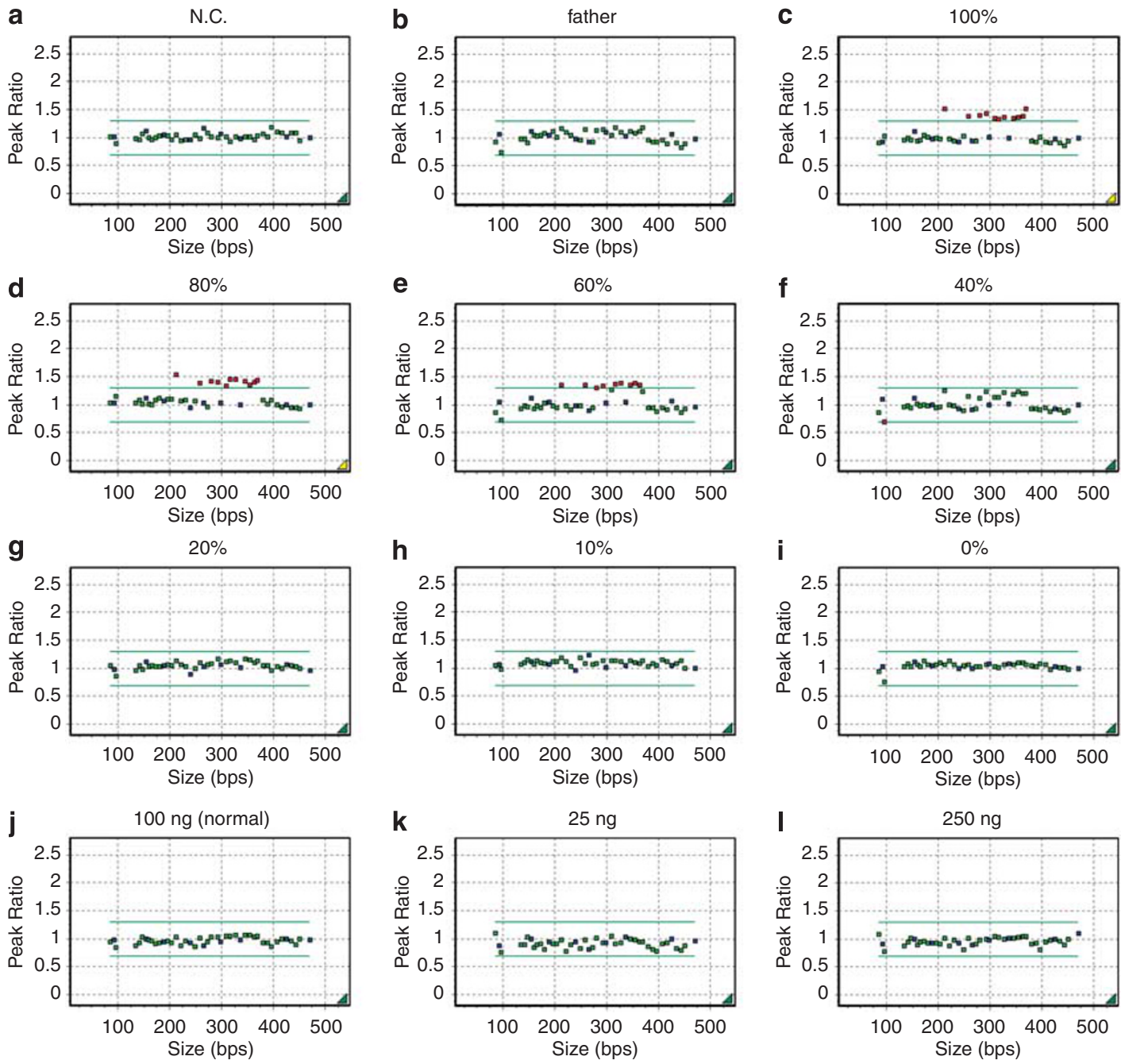

Figure 1 MLPA analysis of TSC2. The graphics showed the MLPA results of peak ratio versus size of (a) a negative control sample, (b) father of the index patient, (c) index patient, (d-i) mixtures carrying the duplication in, respectively, 80 (d), 60 (e), 40 (f), 20 (g), 10 (h) and $0 \%$ of the cells (i); (j-l) father of the index patient with a range of input DNA of, respectively, $100 \mathrm{ng}$ (normal amount) (j), $25 \mathrm{ng}$ (k) and $250 \mathrm{ng}$ (I). A blue dot refers to two copies of an internal control probe, a green dot to two copies of a gene specific probe and a red dot to respectively a deletion or duplication $(<0.70$ or $>1.30)$ of a probe).

the peak results were tested. No differences between the results were seen (data not shown).

Long-range PCR, followed by sequencing of the abnormal product resulted in the identification of pathogenic mutation. The duplication started in intron 14 and extended to the middle of exon 27 of TSC2, combined with an insertion of CACG (c.[3232insCACG;160075_3232dup13997]).

For confirmation, a duplication specific PCR was designed and used for testing the family members. The abnormal fragment of $627 \mathrm{bp}$ and the wild-type fragment of $1315 \mathrm{bp}$ were clearly visible in the index patient (Figure 2, lane 5). The duplication was not detected in control samples but was present in the father (Figure 2, lanes 1-4). However, the ratio of the mutant versus the wild-type fragment was lower compared with the index patient, indicating that the father was somatic mosaic for the mutation, confirming the result of the Southern blot analysis.

To estimate the percentage of affected white blood cells of the father, a titration curve was prepared by mixing DNA of the index patient

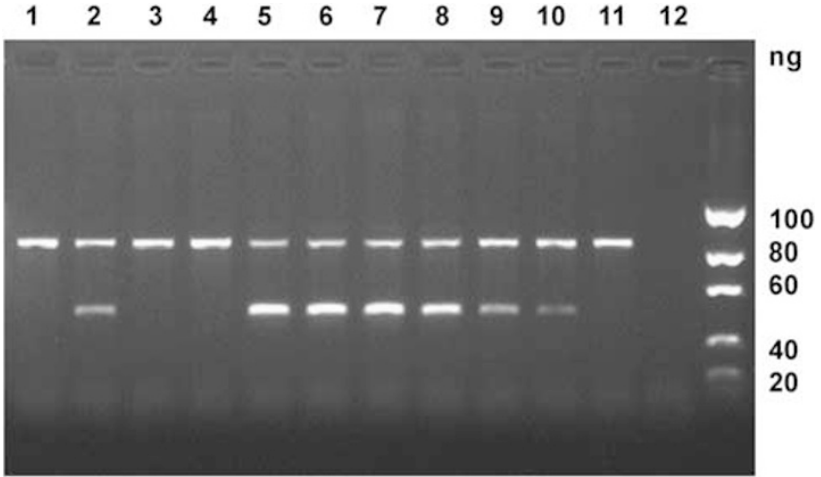

Figure 2 Agarose gel electrophoresis of the TSC2 duplication specific PCR products. Lane 1, normal control; lane 2, father; lane 3 and lane 4, normal control; lane 5, index patient (100\%); lane 6, $80 \%$ mosaic; lane 7, $60 \%$ mosaic; lane 8, 40\% mosaic; lane 9, 20\% mosaic; lane 10, 10\% mosaic; lane $11,0 \%$ mosaic; lane 12 , no DNA. 
with a negative control (Figure 2, lanes 5-11). The duplication-specific PCR showed that about $30 \%$ of the father's leukocytes carried the duplication (Figure 2, lane 2).

The detection rate of the MLPA was compared with that of the duplication-specific PCR using the previously mixed samples. Figure 2 showed that the duplication-specific PCR was able to detect a mosaic pattern of about $10 \%$. A mosaic pattern of about $40 \%$ could be detected by MLPA analysis (Figure 1c-i), which explains why the $30 \%$ of the father could not be detected by MLPA (Figure 1b).

To investigate the sensitivity of the MLPA in detecting deletions, two patients with a total NF1 deletion previously identified using FISH analysis were tested. Patient 19578 carried the deletion in $85.4 \%$ of the cells and patient 26839 in 22\%. MLPA analysis also showed the total gene deletions (Figures $3 \mathrm{a}$ and $\mathrm{b}$ ). The sensitivity of the MLPA kit in detecting this NF1 total gene deletion was determined by a titration analysis using DNA of family 19578. Using the NF1-1 MLPA kit P081, this deletion may be detected when it is present in about $30 \%$ of the cells (Figure 3a). The NF1-2 MLPA kit P082 showed the same sensitivity (data not shown). These analyses showed that it is possible to detect a deletion present in $22-30 \%$ of the cells. These results indicate that, using a quantitative analysis, mosaic duplications are harder to detect than deletions. The size of the rearrangement and the probe density can influence the detection. It can be difficult to identify mosaic duplications or deletions of one exon, as the signal of the probe is also depending of DNA quality. ${ }^{8}$

It has been shown that MLPA analysis can be used to detect highgrade mosaic NF1 patients. ${ }^{6}$ We investigated the sensitivity of the MLPA to detect a duplication of seven exons of TSC2 and total NF1 deletions. Our results indicated that a low percentage of somatic mosaicism could be missed. Somatic mosaicism of a single exon deletion or duplication will be even harder to identify. We recommend to confirm the abnormal MLPA patterns using an alternative technique; it is usually feasible to characterize the breakpoint and to develop a gene defect-specific PCR. Otherwise, for larger rearrangements, especially deletions, FISH analysis can be a reliable technique for the detection of low-grade mosaicism. Depending on probe availability and the identified abnormality, BACs, cosmids or fosmids can be used as a probe. Another possibility, depending on the probe number in the area of interest, is to use an oligonucleotide or SNP-based array. It is known that high-density SNP technology has a detection rate of up to $\sim 30 \%,{ }^{9}$ which makes the sensitivity of this technique comparable with MLPA.

Detection of a low-grade mosaic parent has great implications for genetic counseling. In case the pathogenic mutation can not be detected in DNA of the parents, a low recurrence risk on affected offspring will be given. If somatic mosaicism is identified in one of the

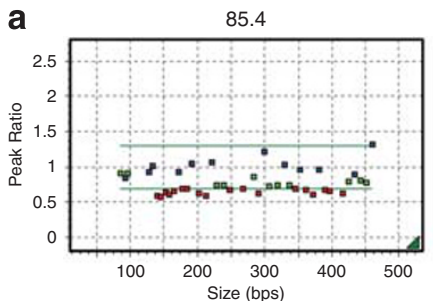

$49 \%$

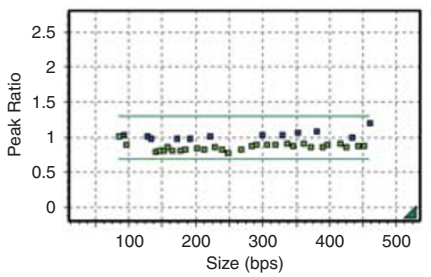

$20 \%$

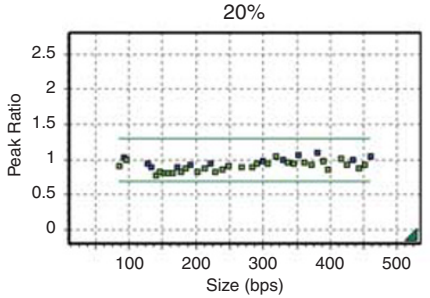

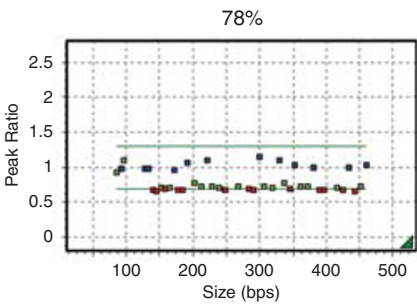

$39 \%$

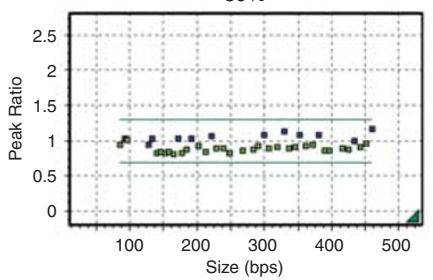

$10 \%$

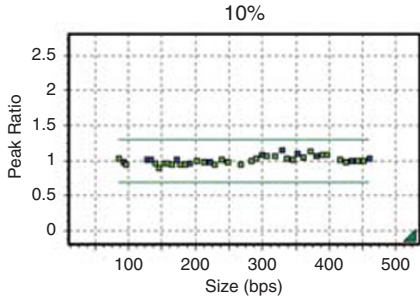

$22 \%$

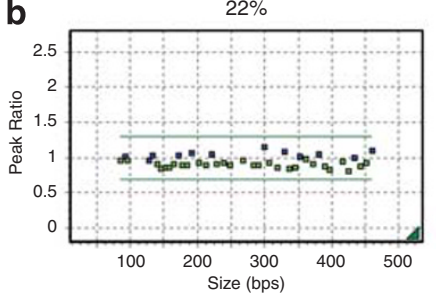

b

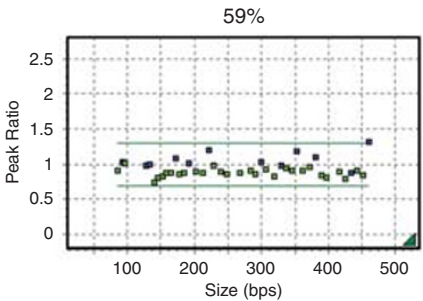

$29 \%$
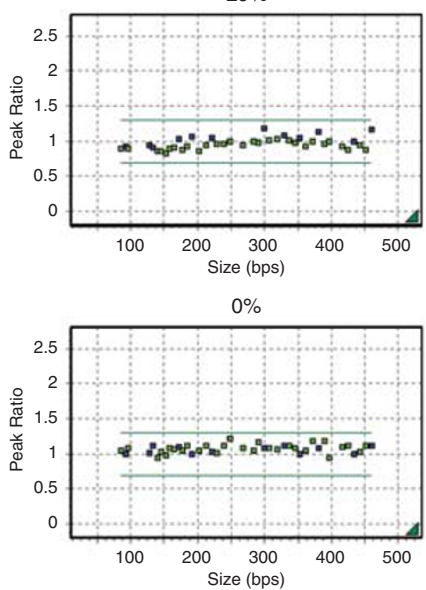
parents, the recurrence risk may be up to $50 \%$, indicating the necessity to use the most sensitive technologies for the analysis of parental DNA.

\section{CONFLICT OF INTEREST}

The authors declare no conflict of interest.

1 Verhoef S, Bakker L, Tempelaars AM et al: High rate of mosaicism in tuberous sclerosis complex. Am J Hum Genet 1999; 64: 1632-1637.

2 van Slegtenhorst $M$, de Hoogt $R$, Hermans $C$ et al: Identification of the tuberous sclerosis gene TSC1 on chromosome 9q34. Science 1997; 277: 805-808.

3 Sancak 0, Nellist M, Goedbloed M et al: Mutational analysis of the TSC1 and TSC2 genes in a diagnostic setting: genotype-phenotype correlations and comparison of diagnostic DNA techniques in tuberous sclerosis complex. Eur J Hum Genet 2005; 13: 731-741.

4 Ferner RE, Huson SM, Thomas $\mathrm{N}$ et al: Guidelines for the diagnosis and management of individuals with neurofibromatosis 1. J Med Genet 2007; 44: 81-88.

5 Kozlowski P, Roberts P, Dabora S et al: Identification of 54 large deletions/duplications in TSC1 and TSC2 using MLPA, and genotype-phenotype correlations. Hum Genet 2007; 121: 389-400.

6 Wimmer K, Yao S, Claes $\mathrm{K}$ et al: Spectrum of single- and multiexon NF1 copy number changes in a cohort of 1,100 unselected NF1 patients. Genes Chromosomes Cancer 2006; 45: 265-276.

7 Nellist M, Sancak O, Goedbloed MA et al: Large deletion at the TSC1 locus in a family with tuberous sclerosis complex. Genet Test 2005; 9: 226-230.

8 van den Ouweland AM, Elfferich P, Zonnenberg BA et al: Characterisation of TSC1 promoter deletions in tuberous sclerosis complex patients. Eur J Hum Genet 2011; 19: 157-163.

9 Peiffer DA, Le JM, Steemers FJ et al: High-resolution genomic profiling of chromosomal aberrations using infinium whole-genome genotyping. Genome Res 2006; 16: 1136-1148. 